\title{
La fécondité des migrantes internes en Belgique \\ Une approche longitudinale et spatiale à partir des données rétrospectives des recensements
}

\author{
THIERRY EGGERICKX ${ }^{1}$ \\ RAfael COSTA ${ }^{1}$ \\ JeAN-PAUL SANDERSON ${ }^{1}$
}

\begin{abstract}
Résumé
Cette étude propose d'une part, une analyse spatiale de l'évolution de la fécondité en Belgique au cours du 20ème siècle et, d'autre part, une étude des effets de la migration sur la fécondité. L'exploitation des données individuelles et rétrospectives des recensements de la population de 1981 et de 2001, nous a permis de reconstituer la vie génésique complète des générations de femmes nées entre le début du 20ème siècle et les années 1960 . Nous avons d'abord testé la pertinence d'une approche rétrospective dans le cadre d'une analyse spatiale de la fécondité. En effet, alors que le lieu de résidence des femmes est défini à la date du recensement, elles ont pu donner naissance ailleurs; dans ce cas, les indicateurs spatiaux de fécondité (par exemple, la descendance finale par commune) calculés par cette approche rétrospective pourraient être biaisés. Néanmoins, les tests de validation que nous avons menés démontrent que ce biais est insignifiant. Ensuite, nous avons analysé l'impact de la migration sur la fécondité, en comparant les comportements de fécondité des migrantes (les femmes qui ont changé de type de commune) et des non-migrantes. Les comportements de fécondité des femmes se conforment plutôt à ceux pratiqués au lieu de destination qu'au lieu d'origine. Les résultats vérifient davantage les hypothèses d'adaptation et de sélection que celle de la socialisation.
\end{abstract}

Mots-clés

Belgique, fécondité, migration, approche spatiale et longitudinale.

1. Centre de recherche en démographie et société, Université catholique de Louvain, Louvain-la-Neuve, Belgique. 


\begin{abstract}
Summary
This study investigates the spatial dimension of fertility evolution in Belgium during the 20th century, and in particular the effect of migration on fertility. Based on the 1981 and 2001 Belgian Censuses data at the individual level, we use a retrospective approach in order to reconstitute complete fertility histories of women born between the early 20th century and the 1960's. The first part of the article tests the relevance of a retrospective approach for spatial analysis. In fact, whereas the women's place of residence is defined at the census date, they could have experienced their childbearing elsewhere; therefore, spatial fertility indicators (e. g. total fertility by municipality) calculated by a retrospective approach could be biased. However, our validation tests show that such bias is irrelevant. In the second part, we investigate the impact of migration on fertility. We compare fertility behaviour of migrants (i. e. women who changed municipality types) and non-migrants. Results suggest that migrants' fertility behaviour is closer to the dominant behaviour at destination than to that at origin, which rejects socialisation hypothesis while supporting the adaptation or selection hypotheses.
\end{abstract}

\title{
Keywords
}

Belgium, fertility, migration, spatial and longitudinal approach

\section{Introduction}

Les travaux consacrés aux différences spatiales de fécondité et à leur évolution en Belgique sont rares², probablement par manque d'intérêt pour les particularités locales des comportements démographiques et surtout faute de données disponibles à une échelle spatiale fine. Dans une optique transversale, on ne dispose en Belgique de données locales de fécondité qu'à partir de 1991. Par contre, l'exploitation des données individuelles, anonymisées et informatisées des derniers recensements de la population permet de reconstituer la vie reproductive complète de générations de femmes nées entre le début du 20ème siècle et le milieu des années 1960 selon le lieu de résidence. C'est sur ces données ré-

2. Citons néanmoins le travail précurseur de Lesthaeghe (1977) sur l'évolution de la fécondité en Belgique de 1800 à 1970, ainsi que l'étude de Lesthaeghe et Neels (2002) sur l'évolution des schémas spatiaux des première et seconde transitions démographiques en Belgique, France et Suisse. Dans le cas de la Belgique, ces travaux sont réalisés à l'échelle des arrondissements. 
trospectives et longitudinales que repose cette étude ${ }^{3}$. Celle-ci se subdivise en quatre parties.

En amont des analyses, nous présentons un bref état des connaissances et les hypothèses de travail, ainsi que les données utilisées et les choix méthodologiques opérés.

La troisième partie s'interroge sur la pertinence, à l'échelle des communes et des sous-régions, d'une approche rétrospective et longitudinale de la fécondité à partir des données des recensements. L'appartenance territoriale des femmes est déterminée par la commune de résidence à la date des recensements. Or, leur vie reproductive a pu se dérouler en partie, voire totalement, hors de ce territoire, compte tenu des migrations effectuées durant cette période. Dans ce cas, il n'y aurait aucun lien ou au mieux une relation indirecte entre le lieu de résidence au recensement et la fécondité des femmes concernées. Quel est l'impact des migrations internes sur les indices longitudinaux et spatialisés de fécondité calculés aux recensements?

Analyser l'influence de la migration sur la fécondité d'un territoire revient aussi à s'interroger sur les comportements de fécondité des migrants : ont-ils des niveaux de fécondité similaires à ceux qui caractérisent l'environnement de leur enfance, là où ils furent «socialisés», ou au contraire, leurs comportements sont-ils semblables à ceux de la population d'accueil ? La quatrième partie compare la fécondité de différents types de migrantes à celle des sédentaires. Observe-t-on des différences significatives entre ces groupes ? Ces différences évoluent-elles au fil des générations ? Se marquent-elles davantage dans certaines régions, sousrégions ou lieu de résidence?

\section{État des connaissances et hypothèses de recherche}

La légitimité de cette étude repose sur une première hypothèse : l'existence, aujourd'hui encore, de différences significatives de comportement de fécondité à l'intérieur d'un petit pays comme la Belgique. Une étude récente (Costa et al., 2010) vérifie cette hypothèse et confirme la présence de «territoires» de fécondité, spatialement homogènes. Relevons

3. Cette recherche se situe dans le prolongement de deux études récentes proposant une analyse spatio-temporelle de la fécondité en Belgique selon des approches transversale (Costa et al., 2010) et longitudinale (Costa el al., 2011). 
très brièvement les principales conclusions de cette étude qui repose sur une analyse transversale de la fécondité.

- Après une phase de convergence des niveaux de fécondité (indices conjoncturels de fécondité) dans les années 1980, les différences entre communes se sont accentuées à partir des années 1990.

- L'analyse de l'évolution de l'indice conjoncturel de fécondité entre 1971-1975 et 2001-2005 met en évidence tantôt des mutations rapides, tantôt des inerties fortement ancrées dans l'histoire. Ainsi, les communes du Nord-Est de la Belgique (Campine), qui se caractérisaient encore vers 1975 par une fécondité très élevée, se distinguent aujourd'hui par les niveaux les plus faibles, de l'ordre de 1,5 enfant par femme (Figure 1). Au contraire, l'Ardenne (Sud de la Belgique) présente, hier comme aujourd'hui, une fécondité élevée (souvent supérieure à 2,1 enfants par femme), et cela en dépit d'une profonde mutation sociodémographique, marquée par une intense attractivité migratoire (Eggerickx et al., 2011).

- La cartographie de l'indicateur de calendrier de la fécondité ${ }^{4}$ (Figure 2) ne correspond pas à celle de la mesure de l'intensité (Figure 1). De nombreux territoires dans le Nord du pays ont des niveaux très bas de fécondité et un calendrier précoce, alors que dans le Sud du pays, les fécondités élevées sont associées à un calendrier précoce. Mais cette mesure de calendrier porte surtout l'empreinte de la périurbanisation : les riches communes périurbaines, autour de Bruxelles et de Namur, au Sud d'Anvers et de Liège, qui se caractérisent par les niveaux d'instruction et les taux d'activité féminine les plus élevés, présentent les calendriers les plus tardifs. Dans ces espaces périurbains, en moyenne moins de $50 \%$ de la fécondité est réalisée avant 30 ans, alors que dans les communes du Nord-Ouest de la Flandre, cette proportion atteint parfois $75 \%$.

Cette approche spatiale et transversale de la fécondité présente plusieurs limites. À l'échelle des communes, il n'existe aucune donnée publiée et seul le traitement des fichiers individuels du Registre national permet de produire des indicateurs de fécondité. Outre les difficultés d'accès à ces bases de données, celles-ci ne sont disponibles qu'à partir de 1991, lorsque le Registre national devint pleinement opérationnel. Ensuite, les indicateurs produits se résument aux indices synthétiques, tels quel l'indice conjoncturel de fécondité ou l'âge moyen à la maternité.

4. Il s'agit de la contribution (en \%) des femmes de moins de 30 ans à l'indice conjoncturel de fécondité, soit la somme des naissances réduites entre 15 et 29 ans. 
FIGURE 1 L'indice conjoncturel de fécondité (ICF-nombre moyen d'enfants par femme) de la population des communes belges de 2001 à 2005

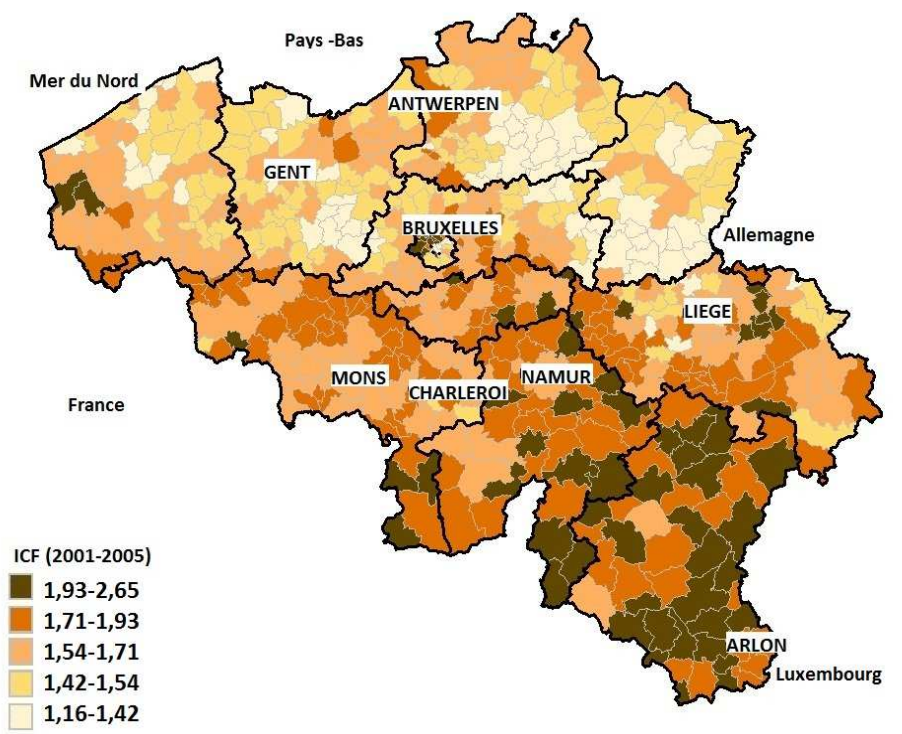

Figure 2 La contribution (en \%) des femmes de moins de 30 ans à l'indice conjoncturel de fécondité de la population des communes belges de 2001 à 2005

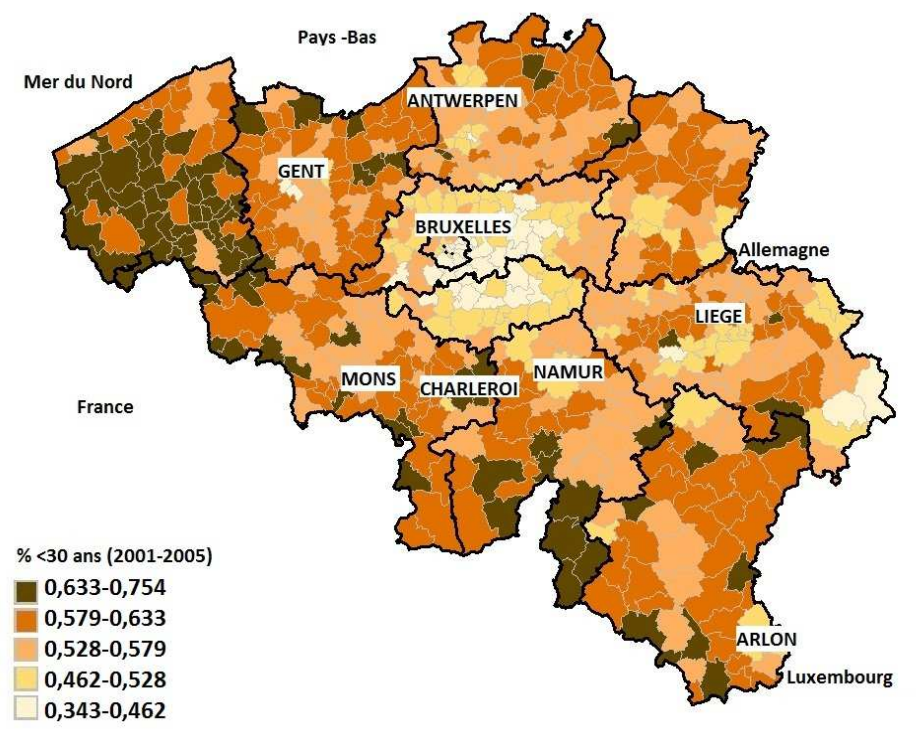

Source des figures 1 et 2 : Registre national, Costa et al., 2010. 
Les données individuelles et informatisées des recensements de la population offrent des perspectives nouvelles pour l'étude de l'évolution de la fécondité depuis le début du 20ème siècle 5 . Elles autorisent une approche longitudinale et rétrospective de la fécondité qui repose sur la production d'indicateurs diversifiés, tels que les descendances finales, les parités, les probabilités d'agrandissement, les intervalles entre naissances, les âges aux maternités, etc. Elles permettent aussi de croiser la fécondité avec des variables socio-économiques (niveau d'instruction, statut d'activité ou occupation des mères, etc.) et de prendre en considération la dimension spatiale du phénomène selon un découpage territorial très fin, puisque l'on dispose de l'exhaustivité de la population. Or, à l'exception d'un article récent de Neels (2010) et de la monographie du recensement de 1991 consacrée à la fécondité (Schoenmaeckers et al., 2002), rares sont les études ayant exploité ces données rétrospectives.

Outre les éventuels effets de sélection induits par les sorties d'observation (par décès et migrations internationales), les erreurs de mémoire et le problème des non-réponses, une approche spatiale de la fécondité à partir des données rétrospectives extraites des recensements de la population se heurte à l'effet perturbateur des migrations internes. En effet, c'est la commune de résidence au recensement qui définit le lieu de résidence des femmes. Or, celui-ci ne correspond pas forcément au(x) lieu(x) de résidence où la femme a eu ses enfants, compte tenu des migrations réalisées pendant et après sa vie génésique. En d'autres termes, la fécondité de ces femmes migrantes ne serait pas forcément conforme aux comportements reproductifs de leur lieu de résidence au recensement.

En fonction des différences spatiales de fécondité du moment (Figures 1 et 2) et de l'importance des migrations internes, la spatialisation de la fécondité, selon une approche rétrospective et longitudinale, semble théoriquement peu crédible. Or, plusieurs arguments plaident le contraire. Tout d'abord, la plupart des migrations, définies comme un changement de commune de résidence, s'effectue sur de petites distances ${ }^{6}$ et n'implique pas forcément un changement de «territoire de fécondité» (Clark, Huang, 2003 ; Michielin, Mulder, 2005). Ensuite, la cartographie

5. Il s'agit des questions posées aux femmes sur le nombre d'enfants nés vivants et leur date de naissance.

6. En moyenne, on dénombre chaque année 1000000 de changements de logement. La moitié se déroule à l'intérieur d'une même commune, alors que l'autre moitié concerne des migrations entre communes. Parmi celles-ci, seules 200000 impliquent un changement d'arrondissement et 65000 un changement de région (Eggerickx et al., 2011). 
communale des descendances finales et âges à la première maternité par génération (Costa et al., 2011) corrobore fidèlement les résultats des analyses transversales et les études sur l'histoire de la fécondité en Belgique (Lesthaeghe, 1977 ; Lesthaeghe, Neels, 2002). Il n'y aurait donc pas de contre-indication à procéder à une étude de la fécondité à l'échelle des communes à partir des données rétrospectives extraites des recensements de la population. On peut dès lors émettre les postulats suivants :

A la présence au recensement, dans un territoire donné, de femmes dont la vie reproductive s'est déroulée partiellement ou totalement ailleurs ne modifie guère la fécondité moyenne du territoire en question. En d'autres termes, l'effet perturbateur des migrations sur la fécondité du lieu est faible ;

B la fécondité des migrantes d'un territoire donné est relativement similaire à celle des non-migrantes du même territoire.

En résumé, nous proposons ici une approche agrégée qui repose sur l'identification, d'une part, de types de communes définis comme des espaces homogènes de fécondité et, d'autre part, de sous-populations définies selon le type de mouvement migratoire réalisé, ou non, au cours d'une période d'observation donnée. La comparaison des indicateurs de fécondité des sédentaires et des migrantes stables (celles qui ont passé la totalité ou une grande partie de leur vie génésique dans la commune de résidence au recensement) avec les indicateurs totaux de chaque type de communes permettra de vérifier la première hypothèse $(A)$ et donc de mesurer l'effet perturbateur des migrations sur la fécondité calculée à partir des données rétrospectives des recensements de la population. L'analyse comparative des indices d'intensité et de calendrier de fécondité des sédentaires et des migrantes stables permettra de tester la seconde hypothèse (B), à savoir la proximité des comportements reproducteurs de ces deux sous-populations.

La littérature scientifique (Kulu, 2005, 2006 ; Kulu, Milewski, 2007 ; Gabrielli et al., 2007) identifie généralement quatre hypothèses concernant les comportements de procréation des migrants :

- Hypothèse de socialisation : les comportements des migrants seraient conditionnés par ceux prévalant dans leur environnement d'enfance. Ils conserveraient donc des comportements proches de ceux de leur milieu d'origine et différents de leur milieu d'accueil.

- Hypothèse d'adaptation: elle suppose que les comportements des migrants se conforment à ceux de la population du nouvel environnement socioculturel et économique. 
- Hypothèse de sélection : les migrants constituent un groupe sélectionné dont les comportements de reproduction diffèrent de ceux de leur société d'origine et se rapprochent de ceux de la société d'accueil. La sélection peut s'opérer sur base de caractéristiques telles que le niveau d'instruction ou le type d'activité, mais aussi de facteurs moins mesurables, comme les projets de mobilité sociale et les prédispositions familiales.

- Hypothèse de perturbation : elle suppose que la migration elle-même va «perturber» le schéma reproductif des migrants. Cette perturbation peut être liée au coût économique de la migration, à la période d'installation dans un nouveau logement ou aux conséquences sociopsychologiques du déménagement (Kulu, 2006) ; un ensemble de facteurs qui peuvent retarder les naissances et donc entraîner temporairement une fécondité moins élevée.

Comme nous supposons une relative adéquation entre les comportements reproducteurs des migrantes et des non-migrantes du lieu d'accueil (hypothèse b), il y aurait donc un effet d'adaptation et/ou de sélection des migrantes.

De nombreux travaux ont également souligné les interactions entre les comportements reproducteurs et les changements de logements, et par extension, le choix du lieu de résidence. Dans le cas de la France, Courgeau (1987) a démontré que les migrantes des espaces ruraux vers les centres urbains se caractérisent par une fécondité plus faible que celle des femmes de leur milieu d'origine et des comportements similaires à ceux des femmes du milieu d'accueil, ce qui révélerait un effet de sélection. En revanche, les migrations de la ville vers la campagne s'accompagneraient d'une augmentation de la fécondité et d'une adaptation des comportements à ceux en vigueur dans les espaces d'accueil. Ces comportements différentiels selon le milieu de destination pourraient aussi être liés à un effet de composition des caractéristiques du logement. En milieu urbain, le parc de logements se compose surtout d'appartements locatifs et de petite taille (Thomas et al., 2011), des caractéristiques qui peuvent contraindre la fécondité. En milieu rural ou périurbain, les logements sont le plus souvent de type unifamilial et de plus grande taille, occupés à titre de propriétaire et disposant d'un jardin (Thomas et al., 2011), ce qui peut favoriser une descendance plus nombreuse (Mulder, 2006). L'étude de Kulu et Vikat (2007) sur la Finlande a démontré que la fécondité des couples propriétaires d'une maison unifamiliale était significativement supérieure à celle des couples vivant en appartement.

Michielin et Mulder (2005) ont structuré ces interactions entre fécondité et «migration de logement» selon deux mécanismes d'ajustement et 
d'anticipation. Le premier suppose que la naissance du ou des premiers enfants précède la migration ; celle-ci serait donc une stratégie d'ajustement des caractéristiques du logement à l'évolution de la composition du ménage et dans ce cas, la migration serait subordonnée à la fécondité. Le second mécanisme suppose que la migration précède la naissance des enfants ; le changement de logement étant une stratégie d'anticipation qui permettrait, compte tenu des caractéristiques du nouveau logement, de concrétiser les objectifs du couple en termes de composition du ménage. Des recherches récentes, menées dans le cas des Pays-Bas (Feijten, Mulder, 2002; Michielin, Mulder, 2005), de l'Allemagne de l'Ouest (Mulder, 2006) ou encore de la Finlande (Kulu, Vikat, 2007) ont démontré que la migration vers un logement unifamilial se réalisait de plus en plus fréquemment avant la naissance des enfants. Cela se traduit généralement par un risque de conception plus élevé chez les migrantes par rapport aux non-migrantes. Plusieurs modèles peuvent coexister (Kulu, Vikat, 2007). Par rapport aux non-migrantes, on peut observer :

- une augmentation du risque de procréer directement après la migration suivie d'une diminution de ce risque, ce qui suppose que la migration est réalisée par des couples qui ont l'intention d'avoir un enfant ;

- le différentiel de fécondité entre migrantes et non-migrantes se marque bien après la migration, ce qui suggère que les nouvelles et meilleures (probablement) conditions de logement favoriseraient la fécondité ;

- une combinaison des deux modèles précédents avec un risque de procréer systématiquement plus élevé chez les migrantes.

Néanmoins, ces relations ne sont pas systématiques. En effet, là où l'offre de logements confortables de grande taille est limitée, où le prix de ces logements est élevé, il peut y avoir concurrence avec le coût d'une descendance plus nombreuse. Cela se traduirait par un retard du calendrier des naissances et/ou une fécondité plus limitée (Mulder, 2006).

\section{Qualité des données et méthodes}

Cette recherche repose sur une base de données individuelles et anonymisées, couplant les informations des recensements de la population de 1991 et de 2001 et du Registre national de 1991 à 2001. 


\section{La qualité des données rétrospectives de fécondité}

extraites du recensement de la population de 2001

Les informations relatives à la fécondité sont extraites du recensement de la population de 2001 grâce aux questions posées aux mères sur le nombre total d'enfants mis au monde et sur leur date de naissance. Il s'agit d'informations rétrospectives qui permettent de reconstituer la vie reproductive de générations de femmes nées entre 1937 et 19667. À priori, la qualité de ces données rétrospectives peut être entachée par des erreurs de mémoire, tant en ce qui concerne le nombre d'événements déclarés que leur calendrier, et par des biais liés aux éventuels effets de sélection induits par la mortalité et la migration (Wunsch, 2001). En d'autres termes, les femmes d'une génération de naissance donnée qui sont sorties d'observation par décès et par émigration internationale avant la date du recensement de 2001 ont-elles ou non les mêmes comportements que celles qui sont restées sous observation ${ }^{8}$ ?

Selon Neels (2010), dont les analyses se basent sur le recensement de 1991, les biais inhérents à l'utilisation de données rétrospectives sont limités et il y a peu de différences par rapport aux informations fournies par l'état civil. La qualité des données rétrospectives de fécondité des recensements de 1961, 1981 et de 2001 a été évaluée dans deux de nos travaux récents (Costa et al., 2011, 2012). Ces évaluations sont basées sur :

- la confrontation des résultats obtenus avec les séries longitudinales existantes et construites à partir d'autres sources de données ;

- la comparaison à générations constantes des résultats extraits des recensements de 1961, 1981 et 2001 ;

- la reconstitution, à partir des informations longitudinales, d'indices transversaux et leur confrontation aux séries existantes ;

- l'analyse de la cohérence spatiale et historique des indices longitudinaux par rapport à l'état des connaissances sur les différences de fécondité du moment, à l'échelle des arrondissements.

7. Il s'agit de générations identifiées à rebours, sur base d'une période d'observation de 5 années, à partir du recensement de 2001. Des tests ont démontré la très bonne fiabilité des données à partir de la génération de femmes nées en 1937 (Costa et al., 2012). La génération née en 1966 est la dernière pour laquelle on dispose d'une vie génésique presque complète, sur base d'un âge moyen à la dernière maternité de 29 ans (Tableau 2).

8. Comme il est très difficile de répondre à cette question, on pose l'hypothèse d'indépendance entre les phénomènes, soit que la mortalité et les migrations internationales n'influent pas sur la fécondité. 
Conformément aux conclusions de Neels (2010), tous les tests menés, tant aux échelles nationale, régionale, qu'à celles des arrondissements et des communes, valident l'usage de ces informations. Les données rétrospectives sur la fécondité extraites des recensements semblent donc particulièrement robustes, conformes aux séries produites par l'état civil et vraisemblablement peu entachées par des biais de mémoire. Quant à l'hypothèse d'indépendance entre les phénomènes démographiques (fécondité, mortalité et migrations), elle est vérifiée dans une très large mesure, et ce, même dans le cadre du découpage spatial en arrondissements ou d'une agglomération urbaine comme Bruxelles, là où les migrations internes sont très importantes.

Les points forts de ces données extraites des recensements concernent donc leur fiabilité, mais aussi leur exhaustivité, ce qui permet de les exploiter dans le cadre d'une approche spatiale de la fécondité.

\section{Typologie des territoires de la fécondité en Belgique}

Comme l'indique Michielin (2002), de nombreuses études sur la fécondité des migrants, tant dans les contextes des pays en développement que des pays industrialisés, se réfèrent aux différences entre milieu rural et milieu urbain. Cette dichotomie classique, souvent imposée par des données d'enquêtes, est trop simpliste pour identifier les variations spatiales de fécondité entre milieux d'origine et de destination ${ }^{9}$. Cela est d'autant plus vrai dans le contexte occidental de périurbanisation où les frontières entre l'urbain et le rural sont de plus en plus floues.

Notre démarche est différente et plus complexe : elle repose sur la construction d'une typologie des territoires de la fécondité en Belgique à partir d'indicateurs transversaux d'intensité et de calendrier de la fécondité observés à l'échelle des 589 communes $^{10}$. Cette typologie, élaborée à partir d'une méthode de classification hiérarchique (méthode de

9. L'étude de Kulu (2006) sur la fécondité des migrants internes en Pologne et en Autriche, basées sur les «Family and Fertility surveys», distingue trois types d'espace : le rural, les petites villes et les grandes villes. L'étude de Courgeau (1987) qui repose sur l'enquête sur la biographie familiale, professionnelle et migratoire de l'INED opère une dichotomie entre les «zones très urbanisées» et les «zones peu urbanisées».

10. L'analyse de classification se base sur 6 indicateurs : 4 indices conjoncturels de fécondité pour les périodes quinquennales 1971-1975, 1981-1985, 1991-1995 et 20012005 et 2 indices de calendrier : la contribution des femmes de moins de 30 ans à la fécondité totale en 1991-1995 et 2001-2005. Les indices calculés pour les périodes 19711975 et 1981-1985 ont été estimés à partir d'indices standardisés de natalité (Costa et al., 2010). 
Ward), a été détaillée dans Costa et al. (2010). Six types de communes ont été identifiés ${ }^{11}$, lesquels se caractérisent par des comportements de fécondité significativement différents. La Figure 3 présente la localisation spatiale de ces groupes et le Tableau 1 leurs principales caractéristiques.

Figure 3 La typologie des communes belges selon les indicateurs transversaux d'intensité et de calendrier de la fécondité

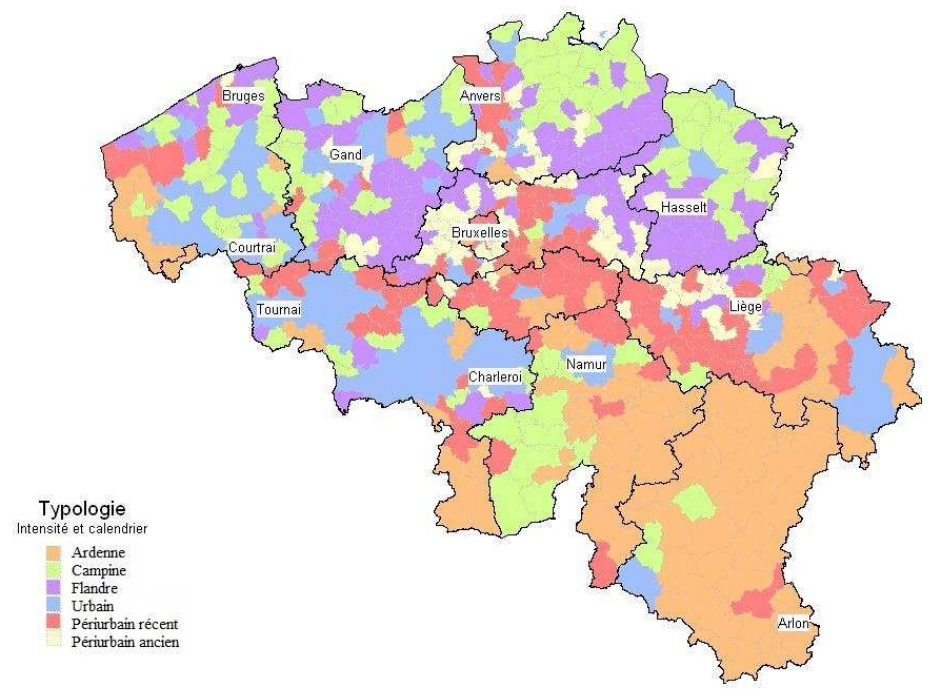

\section{Définition et types de migrants}

Grâce au couplage du recensement de 2001 avec le recensement de 1991 et du Registre national entre 1991 et 2001, nous disposons, pour chaque personne présente en 2001 , du lieu de résidence de sa mère à la naissance, de sa commune de résidence en 1981, 1986, 1991, 1996 et 2001. La principale faiblesse, dans l'état actuel de la base de données et dans le cadre de l'objectif de cette étude, est de ne pouvoir reconstruire la totalité de la biographie migratoire des individus et de ne pouvoir dater avec précision les événements migratoires. En effet, nous identifions

11. Les noms choisis pour les types de communes font référence à leur situation géographique au sens large, sans autre connotation. 
des «migrants résultants» par comparaison des communes de résidence entre $t$ et $t_{+5}$. Nous ne disposons donc d'aucune information sur les éventuelles migrations multiples effectuées au cours de la période d'observation, ni sur la date du changement de commune. Il est donc délicat d'établir des relations de causalité entre les migrations et la fécondité.

TABLEAU 1 Les principales caractéristiques des groupes de communes définis par la typologie

\begin{tabular}{|c|c|c|c|c|c|c|}
\hline $\begin{array}{l}\text { Dénomi- } \\
\text { nation }\end{array}$ & $\begin{array}{c}\text { Nombre } \\
\text { de com- } \\
\text { munes }\end{array}$ & $\begin{array}{c}\text { Type de } \\
\text { communes }\end{array}$ & $\begin{array}{l}\text { Localisa- } \\
\text { tion } \\
\text { régionale }\end{array}$ & $\begin{array}{c}\text { ICF } \\
\text { 1971-75 } \\
\text { (nbr. } \\
\text { enf./fe.) }\end{array}$ & $\begin{array}{c}\text { ICF } \\
2001-05 \\
\text { (nbr. } \\
\text { enf./fe.) }\end{array}$ & $\begin{array}{c}\text { \% de l'ICF } \\
\text { réalisé } \\
\text { avant } \\
30 \text { ans }\end{array}$ \\
\hline $\begin{array}{l}\text { Périurbain } \\
\text { ancien }\end{array}$ & 70 & $\begin{array}{l}\text { Périurbain } \\
\text { très aisé }\end{array}$ & $\begin{array}{l}\text { Flandre- } \\
\text { Bruxelles }\end{array}$ & 1,75 & 1,63 & 52 \\
\hline $\begin{array}{l}\text { Périurbain } \\
\text { récent }\end{array}$ & 116 & $\begin{array}{c}\text { Périurbain } \\
\text { aisé }\end{array}$ & Wallonie & 1,80 & 1,86 & 54 \\
\hline Urbain & 92 & $\begin{array}{l}\text { Urbain } \\
\text { dense }\end{array}$ & $\begin{array}{l}\text { Wallonie- } \\
\text { Flandre }\end{array}$ & 2,10 & 1,74 & 59 \\
\hline Flandre & 105 & «Rural» & Flandre & 2,15 & 1,51 & 57 \\
\hline Campine & 98 & «Rural» & Flandre & 2,50 & 1,66 & 61 \\
\hline Ardenne & 108 & «Rural» & Wallonie & 2,26 & 2,06 & 59 \\
\hline
\end{tabular}

Sur la base des informations disponibles, nous définissons les groupes de femmes suivants :

- Les sédentaires de chaque type de communes.

$\multimap$ Les sédentaires "absolues», soit les femmes qui, aux dates considérées, de la naissance à 2001, n'ont jamais changé de commune.

$\multimap$ Les sédentaires intra-type, soit les femmes qui, aux dates considérées, de la naissance à 2001 n'ont jamais changé de type de communes; elles ont cependant migré entre 1981 et 2001 entre les communes d'un même type.

- Les migrantes «stables» : soit celles qui ont migré d'un type de communes A vers un type de communes B entre leur naissance et 1981, mais qui de 1981 à 2001 sont restées dans le type de communes B. En fonction des générations, ces migrantes auront passé en grande partie ou en totalité leur vie génésique dans le type de communes $B$. Après migration, il s'agit d'un groupe très stable; entre 1981 et 2001, $93 \%$ des femmes de la génération 1952-1956 sont restées dans la même commune du type, et cette proportion est de $87 \%$ pour la génération 1957-1961. 
- Les migrantes inter-types : soit celles dont le type de communes à la naissance ne correspond pas forcément au type de communes en 1981, lequel ne correspond pas au type de communes en 2001. Il s'agit d'une catégorie très instable ou très mobile : pour les générations nées entre 1952 et 1961, plus des deux tiers des femmes ont changé au moins trois fois de type de communes entre 1981 et 2001.

Les sédentaires et les migrantes stables sont les groupes qui contribuent réellement à la fécondité du type de communes identifié au recensement de 2001. Pour les sédentaires, la totalité de leur vie reproductive s'y déroule, alors que pour les migrantes stables, c'est le cas pour une grande partie, voire la totalité de la vie génésique des générations considérées. Plus précisément, il s'agit des générations suivantes :

- la génération née entre 1952 et 1956 ; les migrantes stables ont une vie génésique qui entre 25-30 et 45-50 ans se déroule dans le type de communes d'accueil ;

- la génération née entre 1957 et 1961 ; les migrantes stables ont une vie génésique qui entre $20-25$ et $40-45$ ans se déroule dans le type de communes d'accueil ;

- la génération née entre 1962 et 1966 ; les migrantes stables ont une vie génésique qui entre 15-20 et 35-40 ans se déroule dans le type de communes d'accueil ${ }^{12}$.

En revanche, les migrantes inter-types se caractérisent par une mobilité très importante et par une vie reproductive qui se déroule dans une très large mesure en dehors du type de communes où elles sont recensées en 2001. C'est donc cette catégorie de migrantes qui en théorie entache la pertinence des données rétrospectives de fécondité issues des recensements.

La composition des différents territoires en 2001 selon le type de population

$\mathrm{Au}$ total, les deux sous-populations - sédentaires et migrantes stables qui déterminent réellement la fécondité de chacun des types de communes représentent $73 \%$ des femmes des différentes générations considérées (Figure 4), alors que les migrantes inter-types, dont la vie génésique n'est pas liée au type de communes en 2001, en constituent environ $25 \%$ des différentes générations. Cette composition des générations varie sensiblement d'un type de communes à l'autre, même si sédentaires

12. Pour ces trois générations, l'âge moyen à la première maternité est respectivement de 24,2, 24,9 et 25,5 ans. 
et migrants stables sont toujours majoritairement représentés. C'est dans les espaces périurbains que leur part relative est la plus faible: $65 \%$ dans le périurbain ancien et $61 \%$ dans le périurbain récent. La situation du groupe urbain-industriel est la plus stable, avec notamment $46 \%$ de sédentaires : cette situation peut paraître paradoxale (compte tenu de la mobilité plus importante qui caractérise traditionnellement les espaces urbains), mais elle s'explique par une mobilité intra-type et intra-communale plus intense, car les options résidentielles sont beaucoup plus élevées dans ce milieu de résidence.

Figure 4 La composition des types de communes en 2001 selon les catégories de population (ensemble des générations 1952-1966)

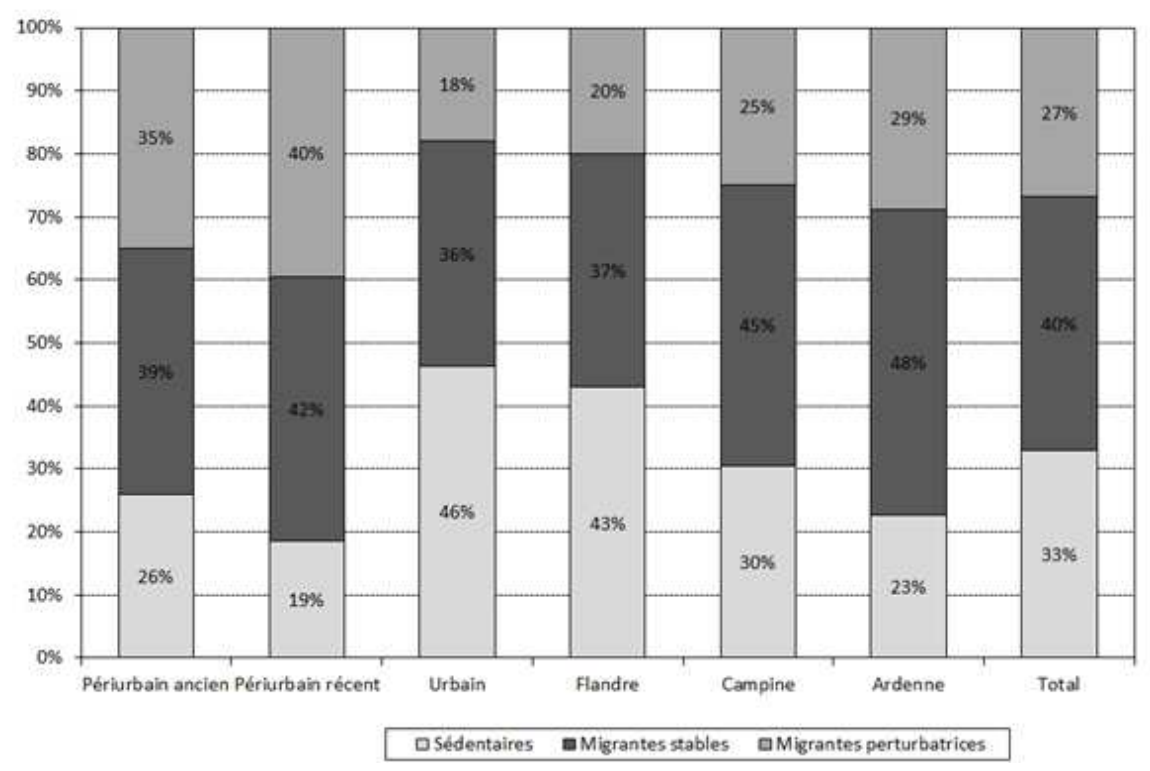

Une approche longitudinale et spatiale de la fécondité est possible à partir de données rétrospectives de recensement

Quel est l'impact réel des migrantes inter-types sur les indices de fécondité - descendance finale, âge moyen à la première maternité, âge moyen à la dernière maternité - des différentes générations observées dans leur type de communes de résidence en 2001 ? Pour répondre à cette question, il suffit de comparer pour chaque génération et chaque type de communes les moyennes totales et les moyennes correspondan- 
tes aux groupes dont la vie reproductive se déroule réellement dans le type de communes en question, soit les sédentaires et les migrantes stables. La différence entre ces moyennes révèlera l'impact de ces migrantes inter-types (Tableau 2).

Compte tenu d'un poids relatif inférieur à celui des sédentaires et des migrantes stables (Figure 4), l'influence de ces migrantes inter-types est minime et se traduit notamment par une très légère surestimation des descendances finales totales pour les générations nées entre 1952 et 1966 et des indicateurs de calendrier pour les générations nées entre 1952 et 1961. Pour la génération 1962-1966, leur impact sur les indicateurs de calendrier est quasiment nul.

TABleaU 2 Indice d'intensité et de calendrier de la fécondité par génération :

l'impact des migrantes inter-types

\begin{tabular}{|c|c|c|c|c|c|c|c|c|c|c|c|c|c|}
\hline \multirow{3}{*}{$\begin{array}{l}\text { Généra- } \\
\text { tions }\end{array}$} & \multirow{3}{*}{ Type commune } & \multicolumn{4}{|c|}{$\begin{array}{l}\text { Descendance finale } \\
\text { (nbr enfants) }\end{array}$} & \multicolumn{4}{|c|}{$\begin{array}{l}\text { Âge première maternité } \\
\text { (années) }\end{array}$} & \multicolumn{4}{|c|}{$\begin{array}{l}\text { Âge dernière maternité } \\
\text { (années }\end{array}$} \\
\hline & & $\mathrm{S}+\mathrm{MS}$ & MIT & Total & Diff. & S+MS & MIT & Total & Diff. & $\mathrm{S}+\mathrm{MS}$ & MIT & Total & Diff. \\
\hline & & (1) & (2) & $(1+2)=3$ & $(3-1)$ & (1) & (2) & $(1+2)=3$ & (3-1) & (1) & (2) & $(1+2)=3$ & $(3-1)$ \\
\hline \multirow[t]{7}{*}{$\begin{array}{l}1952- \\
1956\end{array}$} & otal & 1,89 & 1,92 & 1,90 & 0,01 & 23,9 & 25,3 & 24,2 & 0,3 & 27,9 & 29,6 & 28,3 & 0,4 \\
\hline & Périurbain ancien & 1,74 & 1,81 & 1,76 & 0,02 & 24,5 & 26,0 & 25,0 & 0,5 & 28,1 & 30,1 & 28,8 & 0,6 \\
\hline & Périurbain récent & 1,88 & 1,92 & 1,89 & 0,02 & 24,1 & 25,2 & 24,5 & 0,4 & 28,4 & 29,5 & 28,8 & 0,4 \\
\hline & Urbain & 1,90 & 1,95 & 1,91 & 0,01 & 23,8 & 25,2 & 24,0 & 0,2 & 28,0 & 29,7 & 28,2 & 0,3 \\
\hline & Flandre & 1,76 & 1,85 & 1,78 & 0,02 & 23,9 & 25,2 & 24,1 & 0,2 & 27,3 & 29,2 & 27,6 & 0,3 \\
\hline & Campine & 1,99 & 1,99 & 1,99 & 0,00 & 23,7 & 25,0 & 23,9 & 0,3 & 27,7 & 29,4 & 28,0 & 0,4 \\
\hline & Ardenne & 2,17 & 2,10 & 215 & $-0,02$ & 23,7 & 25,1 & 240 & 03 & 28,6 & 30,3 & 29,0 & 0,4 \\
\hline \multirow[t]{7}{*}{$\begin{array}{l}1957- \\
1961\end{array}$} & 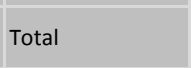 & 185 & 19 १ & 180 & 0,04 & 245 & 25,6 & 240 & 04 & 28,5 & 297 & 289 & 0,4 \\
\hline & Périurba & 1,70 & 1,86 & 1,77 & 0,07 & 25,2 & 26,1 & 25,6 & 0,4 & 28,9 & 30,0 & 29,4 & 0,5 \\
\hline & Périurba & 1,86 & 1,95 & & 0,04 & 24,7 & & 25,2 & 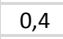 & 29,0 & 29,9 & 29,5 & 4 \\
\hline & Urbain & 1,86 & 1,97 & 1,89 & 0,03 & 24,4 & 25,6 & 24,7 & 0,3 & 28,5 & 29,9 & 28,9 & 0,3 \\
\hline & Flandre & 1,71 & 1,90 & 1,76 & 0,05 & 24,6 & 25,4 & 24,8 & 0,2 & 28,0 & 29,1 & 28,3 & 0,3 \\
\hline & Campine & 1,92 & 2,01 & 1,95 & 0,03 & 24,2 & 25,1 & 24,5 & 0,3 & 28,1 & 29,1 & 28,4 & 0,3 \\
\hline & Ardenne & 2,12 & 2,13 & 2,12 & 0,00 & 24,1 & 25,4 & 24,5 & 0,4 & 29,1 & 30,4 & 29,5 & 0,4 \\
\hline \multirow[t]{7}{*}{$\begin{array}{l}1962- \\
1966\end{array}$} & Total & 1,68 & 1,89 & 1,75 & 0,07 & 25,6 & 25,4 & 25,5 & $-0,1$ & 29,1 & 29,2 & 29,1 & 0,0 \\
\hline & Périurbain ancien & 1,53 & 1,10 & & 0,10 & 26,5 & $\angle 6,0$ & 20,3 & $-0,3$ & 29,6 & 29,5 & 29,5 & $-0,1$ \\
\hline & Périurbain récent & 1,67 & 1,86 & 1,76 & 0,09 & 25,8 & 25,6 & 25,7 & $-0,1$ & 29,4 & 29,5 & 29,4 & 0,0 \\
\hline & Urbain & 1,72 & 1,91 & 1,77 & 0,05 & 25,3 & 25,4 & 25,4 & 0,0 & 29,0 & 29,2 & 29,0 & 0,1 \\
\hline & Flandre & 1,57 & 1,82 & 1,63 & 0,06 & 25,9 & 25,4 & 25,7 & $-0,1$ & 28,9 & 28,7 & 28,8 & $-0,1$ \\
\hline & Campine & 1,75 & 2,00 & 1,83 & 0,07 & 25,4 & 24,7 & 25,2 & $-0,2$ & 28,8 & 28,5 & 28,7 & $-0,1$ \\
\hline & Ardenne & 1,89 & 2,05 & 1,95 & 0,06 & 25,0 & 25,0 & 25,0 & 0,0 & 29,2 & 29,4 & 29,3 & 0,1 \\
\hline
\end{tabular}

$\mathrm{S}+\mathrm{MS}$ = sédentaires et migrantes stables. MIT = migrantes inter-types.

Ces migrantes inter-types se distinguent pour chaque type de communes et chaque génération par une descendance finale légèrement supérieure à celle des sédentaires et des migrantes stables. Pour les générations 1952-1961, elles se caractérisent aussi par un calendrier de la fé- 
condité un rien plus tardif. De plus, assez paradoxalement - puisque leur vie génésique ne s'est pas déroulée dans leur type de communes de résidence de 2001 - les comportements de cette sous-population correspondent à ceux des groupes qui déterminent réellement la fécondité de ce type de communes. Ainsi, par exemple, là où la fécondité est la plus élevée (Campine et Ardenne), les migrantes inter-types se distinguent également par une fécondité élevée. Et inversement, dans les types de communes où la fécondité est la plus basse (Flandre et périurbain ancien), ces migrantes se caractérisent aussi par des niveaux globalement plus faibles. Les mêmes tendances concernent les indicateurs de calendrier.

En conclusion, le biais introduit par ces migrantes inter-types est faible et ne suffit nullement à invalider l'utilisation des données rétrospectives des recensements pour une approche spatiale et longitudinale de la fécondité.

\section{La fécondité des migrantes et des sédentaires}

\section{Des descendances finales assez proches}

Cette analyse se basera essentiellement sur les groupes de femmes dont la vie génésique se déroule totalement ou en grande partie dans le type de communes déterminé au recensement de 2001, soit :

- les sédentaires ;

- les migrantes stables.

Les différences de descendance finale entre les sédentaires et les migrantes stables se chiffrent au maximum à un écart de 0,3 enfant, au profit de ces dernières (Tableau 3). Dans un contexte de basse fécondité, ces écarts peuvent être significatifs d'autant qu'ils sont systématiques. En effet, pour chaque génération et quel que soit le type de communes, les migrantes stables ont toujours une fécondité supérieure à celle des sédentaires. C'est en Ardenne que les différences sont les plus marquées, et en Flandre qu'elles sont les plus réduites.

$\mathrm{Au}$ sein du groupe des sédentaires, les mêmes oppositions différencient les sédentaires absolues - celles qui n'ont jamais changé de commune de résidence depuis leur naissance - des sédentaires intra-type. Ces dernières, quelle que soit la génération et le type de communes, ont toujours une fécondité supérieure à celle des sédentaires absolues. En 
d'autres termes, la sédentarité résidentielle se traduit par une fécondité plus basse, alors que la mobilité résidentielle, à l'intérieur, comme entre types de communes, s'accompagne d'une fécondité plus élevée.

TABleaU 3 La descendance finale par génération et type de communes : comparaison entre les sédentaires et les migrants

\begin{tabular}{|c|c|c|c|c|c|}
\hline Générations & Type de communes & $\begin{array}{c}\text { Sédentaires } \\
\text { absolus } \\
\text { A }\end{array}$ & $\begin{array}{c}\text { Migrantes } \\
\text { intra-type } \\
\text { B }\end{array}$ & $\begin{array}{l}\text { Sédentaires } \\
\qquad A+B\end{array}$ & $\begin{array}{c}\text { Migrantes } \\
\text { stables } \\
\text { C }\end{array}$ \\
\hline \multirow[t]{6}{*}{$1952-1956$} & Périurbain ancien & 1,61 & 1,70 & 1,66 & 1,78 \\
\hline & Périurbain récent & 1,68 & 1,76 & 1,71 & 1,94 \\
\hline & Urbain & 1,74 & 1,88 & 1,79 & 2,00 \\
\hline & Flandre & 1,68 & 1,74 & 1,71 & 1,81 \\
\hline & Campine & 1,84 & 1,98 & 1,90 & 2,04 \\
\hline & Ardenne & 1,80 & 2,11 & 1,92 & 2,26 \\
\hline \multirow[t]{6}{*}{$1957-1961$} & Périurbain ancien & 1,58 & 1,70 & 1,64 & 1,74 \\
\hline & Périurbain récent & 1,72 & 1,81 & 1,76 & 1,90 \\
\hline & Urbain & 1,74 & 1,88 & 1,79 & 1,95 \\
\hline & Flandre & 1,65 & 1,73 & 1,69 & 1,73 \\
\hline & Campine & 1,82 & 1,96 & 1,88 & 1,95 \\
\hline & Ardenne & 1,81 & 2,12 & 1,94 & 2,20 \\
\hline \multirow[t]{6}{*}{$1962-1966$} & Périurbain ancien & 1,47 & 1,52 & 1,50 & 1,56 \\
\hline & Périurbain récent & 1,60 & 1,68 & 1,63 & 1,69 \\
\hline & Urbain & 1,64 & 1,78 & 1,69 & 1,78 \\
\hline & Flandre & 1,53 & 1,58 & 1,55 & 1,59 \\
\hline & Campine & 1,73 & 1,83 & 1,77 & 1,74 \\
\hline & Ardenne & 1,68 & 1,96 & 1,81 & 1,94 \\
\hline
\end{tabular}

Comment interpréter ces différences de comportements qui apparaissent systématiquement au travers des générations et des types de communes? Pour les sédentaires absolues, le choix du logement et de ses caractéristiques serait forcément plus limité et influencerait négativement leur fécondité. Quant aux migrants, l'éventail des types et tailles du logement serait plus étendu et le choix résidentiel effectué en fonction du nombre d'enfants souhaités. Dans ce cas, les caractéristiques du logement ne seraient pas un obstacle à la fécondité (Michielin, 2002). La sédentarité peut être un choix délibéré comme une option contrainte, faute de moyens financiers pour accéder à des logements plus conformes au projet de taille familiale, ce qui induirait alors un effet de sélection sociale au bénéfice des migrants. C'est ce que l'on observe notam- 
ment dans les espaces périurbains et ruraux, où les migrants se caractérisent généralement par des niveaux de vie supérieurs aux sédentaires (Eggerickx et al., 2002 ; Costa et al., 2011).

Par ailleurs, là où la fécondité des sédentaires est élevée, celle des migrantes l'est également et lorsque la fécondité des sédentaires est basse, celle des migrantes suit la même tendance (Figure 5). Ainsi, pour toutes les générations, les sédentaires et les migrantes de «l'Ardenne» se caractérisent par les fécondités les plus élevées. De même, pour les groupes "périurbain ancien» et «Flandre», les deux populations se distinguent par des descendances finales plus faibles qu'ailleurs. Les comportements de reproduction des migrantes stables se conforment donc à ceux qui prévalent dans le type de communes d'accueil. Ce résultat est également celui observé par Kulu (2006) dans le cas de la fécondité des migrants internes en Autriche et en Pologne ainsi que par Michielin (2002) pour les migrants qui quittent l'agglomération de Turin.

L'analyse peut être affinée en distinguant cette fois, pour un type de communes donné, le type de communes d'origine des migrantes stables (Figure 6). Prenons le cas de l'Ardenne, dont la descendance finale des femmes de la génération 1957-1961 est de 2,12 enfants, soit le niveau le plus élevé parmi les types de communes (Tableau 2). Quelle que soit leur provenance, les migrantes se caractérisent par une descendance finale très largement supérieure à celle des sédentaires du type de communes d'origine et conforme à la moyenne du type de communes d'accueil. Le cas du type de communes «Flandre» est tout aussi révélateur. La descendance finale de la génération 1957-1961 y est faible, en moyenne de 1,71 enfant par femme et là aussi, les migrantes stables, peu importe le type de communes de provenance, ont une fécondité faible, inférieure généralement à celle de leurs homologues sédentaires. Cette situation, qui peut être dupliquée pour les autres types de communes et les autres générations, démontre indéniablement que les comportements de reproduction des migrantes se conforment à la situation en vigueur dans le milieu d'accueil. Kulu (2006), dans son étude sur les migrants internes en Pologne et en Autriche, indique aussi que dans les deux pays, la fécondité des migrants ressemble à la fécondité du milieu de destination, et ce, quelle que soit leur origine.

À ce stade de l'analyse, nous ne pouvons toutefois certifier que cette proximité de comportements de reproduction entre sédentaires et migrantes stables reflète davantage un effet de sélection des migrantes qu'un effet d'adaptation à la situation - conditions socio-économiques, caractéristiques des logements, normes socioculturelles - du territoire d'accueil. Mais, en aucun cas, il n'y a un effet de socialisation chez les 

et spatiale à partir des données rétrospectives des recensements

migrantes, qui suppose que les migrantes conservent les comportements de leurs milieux d'origine.

Figure 5 L'évolution de la descendance finale par génération, type de population et type de communes

Génération 1957-1961

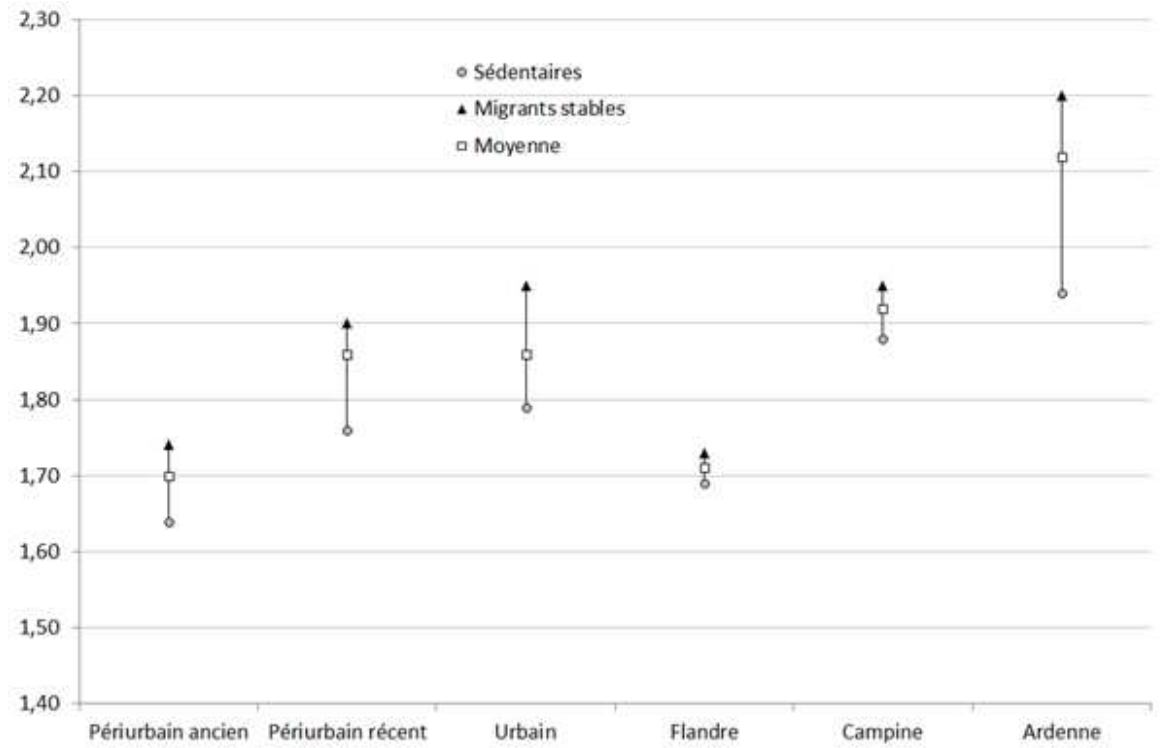

Génération 1962-1966

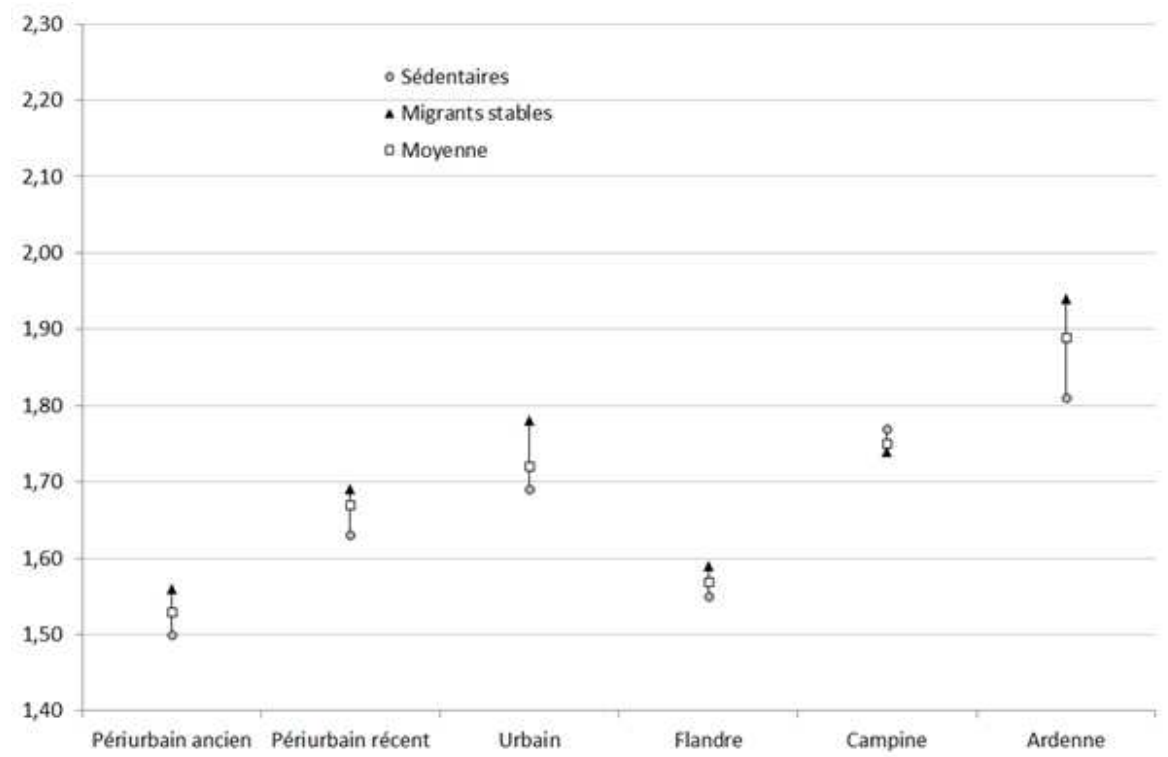


Figure 6 La descendance finale des migrantes stables de la génération 1957-1961 en fonction du type de communes d'origine

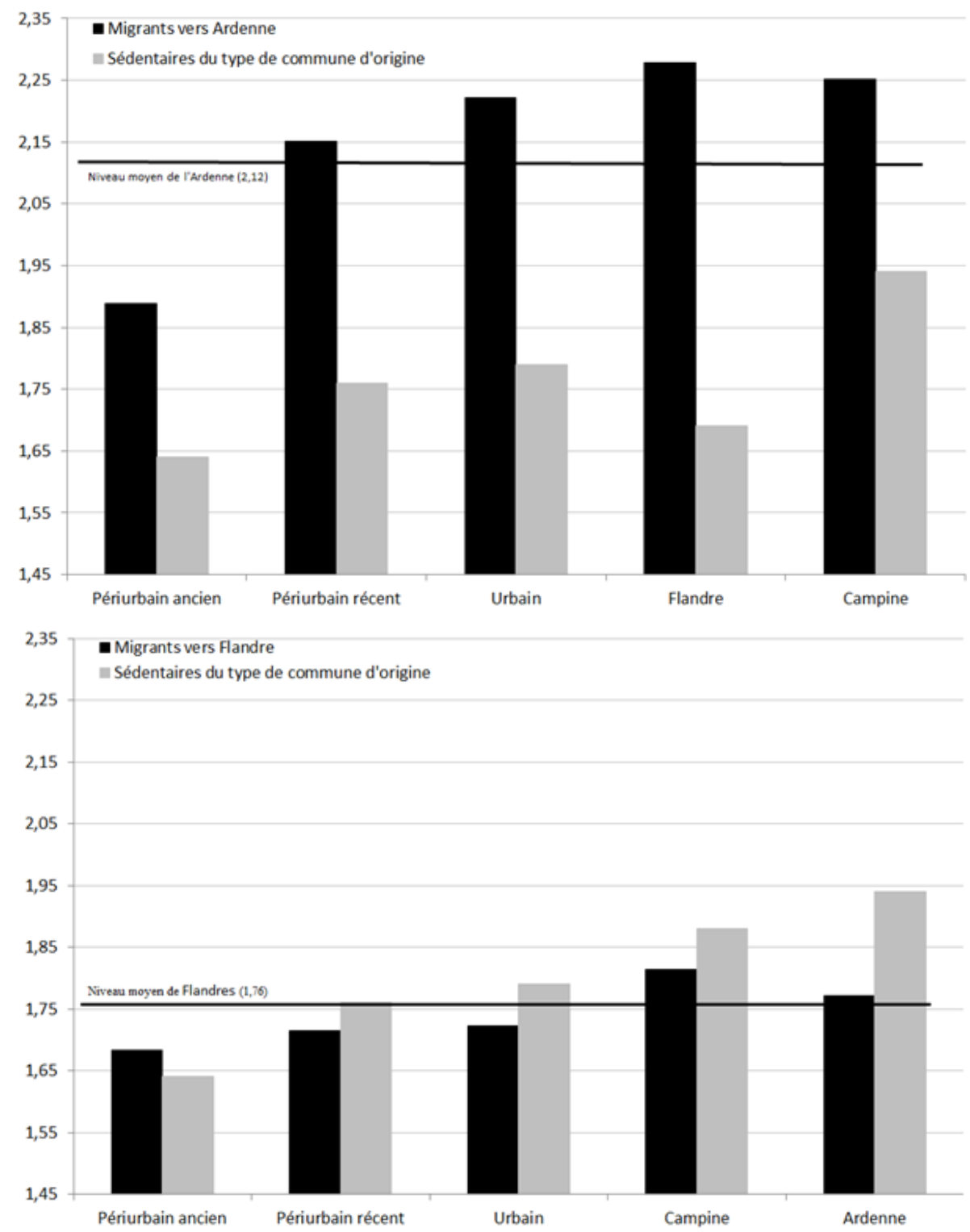




\section{Un calendrier de fécondité plus précoce chez les migrants stables}

Le Tableau 4 présente l'âge moyen à la première maternité par génération et pour les différents types de communes. Pour tous les types de communes, les migrantes stables se caractérisent par un âge moyen à la première naissance un peu plus précoce que celui des sédentaires. Néanmoins, pour la dernière génération (1962-1966), les écarts entre les deux types de population sont insignifiants. Ce résultat rejette a priori l'hypothèse de perturbation qui postule un retard de calendrier chez les migrants dû notamment à la migration et à la période d'installation dans un nouvel environnement. Au-delà de ces différences, l'analyse de cet indicateur de calendrier de la fécondité vérifie une nouvelle fois que les comportements des migrantes stables ressemblent à ceux qui prévalent dans le type de communes d'accueil. En effet, là où l'âge moyen à la première maternité est le plus élevé - périurbains ancien et récent - les migrantes stables se caractérisent également par un calendrier plus vieilli. Et lorsque la première naissance survient plus rapidement - Campine et Ardenne -, les migrantes adoptent le même type de comportement (Tableau 4).

Les différences entre les sédentaires absolues et les migrantes intra-type sont très faibles, sauf dans le cas du «périurbain ancien» où ces dernières accusent un retard de calendrier qui varie de 0,5 à 1,2 année selon la génération. Dans ce milieu de résidence très attractif depuis les années d'après-guerre, où le prix des maisons et des terrains à bâtir a explosé au cours de ces dernières décennies, l'accès au logement est devenu difficile, notamment pour les générations post baby-boom (Eggerickx et al., 2002).

La comparaison des taux de fécondité par âge entre les types de populations conforte ces résultats (Figure 7). Le cas de trois milieux de résidence est illustré ici : périurbain récent, Flandre et Ardenne. Les migrantes stables se démarquent des autres populations par une surfécondité avant 25 ans, et ce, quelle que soit la génération de naissance. Les différences sont très marquées en milieu périurbain et en Ardenne, mais beaucoup plus faibles en Flandre, où d'une manière générale, les comportements reproducteurs des sédentaires et des migrantes sont quasi identiques. Enfin, alors qu'à tous les âges, la fécondité des sédentaires absolues est la plus faible, les sédentaires intra-type se caractérisent par un calendrier plus tardif. 
TABLEAU 4 L'âge moyen à la première maternité par génération et type de communes : comparaison entre les sédentaires et les migrantes

\begin{tabular}{|c|c|c|c|c|c|}
\hline Générations & Type de communes & $\begin{array}{c}\text { Sédentaires } \\
\text { absolues } \\
\text { A }\end{array}$ & $\begin{array}{c}\text { Migrantes } \\
\text { intra-type } \\
\text { B }\end{array}$ & $\begin{array}{c}\text { Sédentaires } \\
\qquad A+B\end{array}$ & $\begin{array}{c}\text { Migrantes } \\
\text { stables } \\
\text { C }\end{array}$ \\
\hline \multirow[t]{6}{*}{ 1952-1956 } & Périurbain ancien & 24,1 & 25,3 & 24,7 & 24,5 \\
\hline & Périurbain récent & 24,1 & 24,5 & 24,2 & 24,1 \\
\hline & Urbain & 23,9 & 24,2 & 24,0 & 23,6 \\
\hline & Flandre & 23,9 & 24,1 & 23,9 & 23,8 \\
\hline & Campine & 23,8 & 23,8 & 23,8 & 23,6 \\
\hline & Ardenne & 24,0 & 24,0 & 24,0 & 23,5 \\
\hline \multirow[t]{6}{*}{ 1957-1961 } & Périurbain ancien & 25,0 & 25,9 & 25,5 & 25,0 \\
\hline & Périurbain récent & 24,9 & 25,1 & 25,0 & 24,6 \\
\hline & Urbain & 24,7 & 24,6 & 24,7 & 24,0 \\
\hline & Flandre & 24,7 & 24,7 & 24,7 & 24,5 \\
\hline & Campine & 24,4 & 24,4 & 24,4 & 24,0 \\
\hline & Ardenne & 24,7 & 24,5 & 24,6 & 23,8 \\
\hline \multirow[t]{6}{*}{ 1962-1966 } & Périurbain ancien & 26,3 & 26,8 & 26,6 & 26,5 \\
\hline & Périurbain récent & 25,7 & 25,7 & 25,7 & 25,8 \\
\hline & Urbain & 25,6 & 25,4 & 25,5 & 25,0 \\
\hline & Flandre & 26,0 & 25,8 & 25,9 & 25,9 \\
\hline & Campine & 26,6 & 25,3 & 25,5 & 25,4 \\
\hline & Ardenne & 25,6 & 24,9 & 25,3 & 24,9 \\
\hline
\end{tabular}

Comme évoqué plus haut, ces différents modèles de fécondité, que l'on retrouve systématiquement pour tous les types de communes et pour chaque génération, pourraient traduire un effet différencié d'anticipation du changement de logement aux souhaits de fécondité. Conformément aux recherches menées dans le cas des Pays-Bas, (Miechelin, Mulder, 2005), de l'Allemagne (Mulder, 2006) et de la Finlande (Kulu, Vikat, 2007), lorsque la migration précède la naissance du premier enfant - ce qui est généralement le cas des migrantes stables -, cela se traduit par une fécondité plus élevée à tous les âges. La migration vers un autre type de communes serait un moyen d'optimiser l'adéquation entre les caractéristiques du logement (taille et type de logement, commodités, environnement) et la descendance souhaitée. En d'autres termes, la rupture avec le type de communes d'origine permettrait d'élargir le choix des possibles en termes de condition de logement, ce qui favoriserait la fécondité. L'option de migrer au sein du même milieu d'habitat - le cas des sédentaires intra-type - rend cette quête du logement idéal plus 
complexe, ce qui se traduit par un report de la première naissance et donc un calendrier décalé. Enfin, pour ceux qui choisissent de rester dans la même commune (les sédentaires), les opportunités de logement optimal sont encore plus réduites, avec pour conséquence une fécondité plus faible et plus tardive.

Figure 7 Les taux de fécondité par âge selon le type de population, la génération de naissance et le type de communes

Périurbain récent :

génération 1952-1956

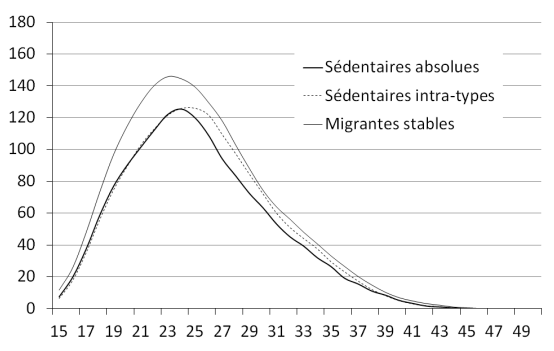

Flandre : génération 1952-1956

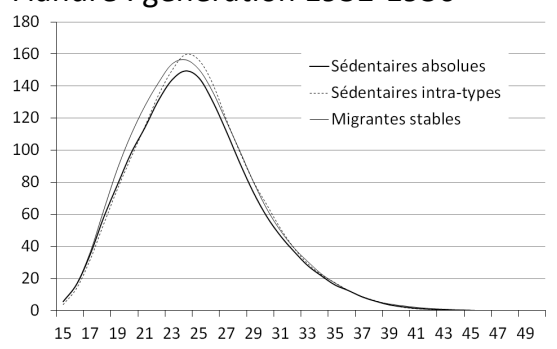

Ardenne : génération 1952-1956

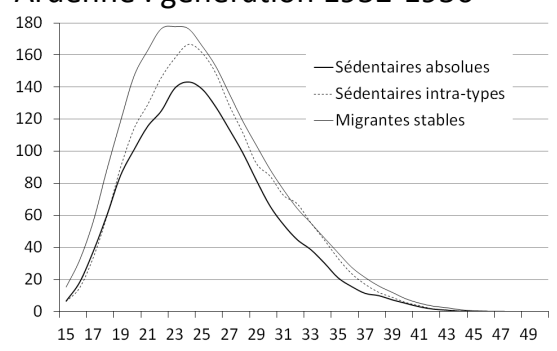

Périurbain récent : génération 1957-1961

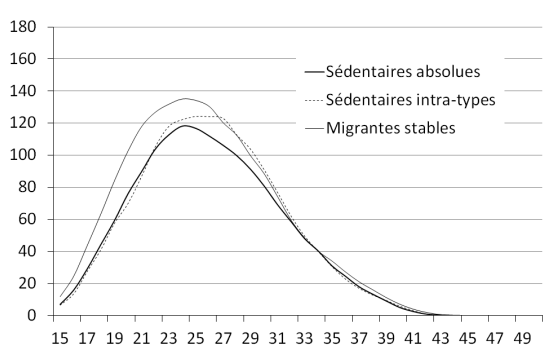

Flandre : génération 1957-1961

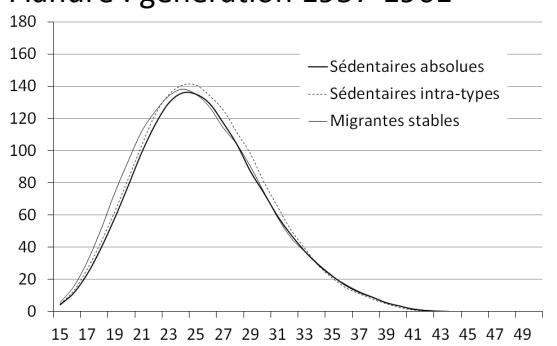

Ardenne : génération 1957-1961

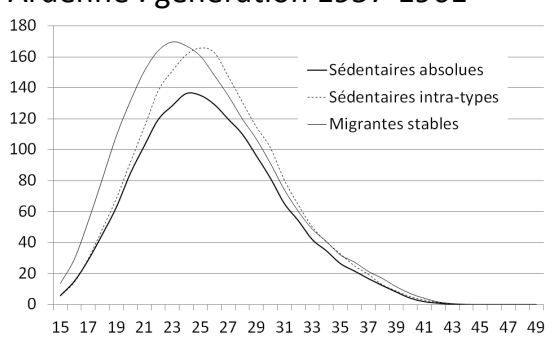




\section{Conclusion}

Les travaux consacrés aux différences spatiales de fécondité et à leur évolution sont rares, en grande partie faute de données adéquates. Nous disposons des données individuelles et anonymisées des recensements de la population de 1981 et de 2001, lesquelles permettent de reconstituer la vie reproductive complète de générations de femmes nées entre le début du 20ème siècle et le milieu des années 1960. C'est sur ces données rétrospectives et longitudinales que repose cette étude.

Nous nous sommes d'abord interrogés sur la pertinence, à l'échelle de communes ou de milieux de résidence, d'une approche rétrospective et longitudinale de la fécondité à partir des données des recensements. C'est la date du recensement qui fixe l'appartenance des femmes à un «territoire» donné. Or, la vie génésique d'une partie de ces femmes a pu se dérouler en partie, voire totalement, hors de ce territoire, compte tenu des migrations effectuées durant cette période. En d'autres termes, cette situation introduirait un biais, car la fécondité de ces femmes ne serait pas forcément représentative de celle du lieu où elles sont recensées. Les analyses que nous avons menées démontrent que ce n'est pas le cas ; l'impact de ces «migrantes perturbatrices» sur la fécondité du type de communes d'accueil calculée au recensement est minime. Dans le cas de la Belgique, il n'y a donc pas de contre-indication à utiliser des données rétrospectives des recensements pour une approche spatiale et longitudinale de la fécondité.

Le deuxième objectif de cette étude était d'analyser les comportements reproductifs des migrants en regard des quatre hypothèses explicatives classiques de socialisation, d'adaptation, de sélection et de perturbation. Plusieurs types de communes ont été distingués et pour chacun d'eux, nous avons confronté les comportements des sédentaires à ceux des migrantes stables qui ont passé une grande partie de leur vie reproductive dans le type de communes en question. Les résultats montrent que, pour chaque type de communes, et pour toutes les générations de femmes considérées, le comportement des migrantes est conforme à celui du territoire d'accueil.

Néanmoins, pour chaque type de communes et pour chaque génération, les migrantes se distinguent par une fécondité plus élevée que celle des sédentaires, et le plus souvent par un âge plus précoce à la maternité. Il pourrait y avoir adaptation de la fécondité des migrantes à la situation du type de communes d'accueil. Mais on ne peut pas non plus exclure un effet de sélection des migrantes, ni un effet d'anticipation des choix rési- 
dentiels et des caractéristiques du logement à la fécondité souhaitée. Par contre, toutes les analyses infirment l'hypothèse de socialisation des migrantes. Enfin, dans nos travaux ultérieurs, il conviendra de tenir compte du niveau socio-économique (activité ou non, type d'activité, niveau d'instruction...) des femmes afin de mieux cerner les effets de sélection et d'adaptation.

\section{Références}

Clark W., Huang Y. (2003), "The Life Course and Residential Mobility in British Housing Markets», Environment and Planning, 35, pp. 323-339, http://dx.doi.org/ 10.1068/a3542.

Costa R., Eggerickx T., Rizzı E., SANderson J.-P. (2010), "Analyse spatiale et temporelle de la fécondité en Belgique: une approche communale», 15ème Colloque national de démographie, CUDEP, Strasbourg, 25-28 mai 2010 (à paraître).

Costa R., Eggerickx T., SANDerson J.-P. (2011), «Les territoires de la fécondité en Belgique au 20ème siècle. Une approche longitudinale et communale», Espaces, Population, Sociétés, 2, pp. 353-375, http://dx.doi.org/10.4000/eps.4550.

Costa R., Eggerickx T., SANDERSON J.-P. (2012), «Les données individuelles et rétrospectives des recensements de la population. Apports et limites pour une approche longitudinale de la fécondité en Belgique de la fin du 19ème siècle à 1970», Communication présentée à la journée d'étude organisée sous l'égide de la Société internationale de démographie historique, Sexualité et contrôle des naissances dans I'histoire (19ème-20ème) : approches quantitatives et qualitatives, 23 mars, Université de Fribourg, $17 \mathrm{p}$.

Courgeau D. (1987), «Constitution de la famille et urbanisation», Population, 1, pp. 57-82, http://dx.doi.org/10.2307/1532757.

Eggerickx T., Capron C., Hermia J.-P., Oris M. (2002), Démographie et développement durable. Migrations et fractures socio-démographiques en Wallonie (19902000), Services Fédéraux des Affaires Scientifiques, Techniques et Culturelles (SSTC), Liège-Louvain-la-Neuve, 208 p. + 32 planches de cartes.

Eggerickx T., Oris M., SANDerson J.-P., VilPert S. (2011), L'évolution de la population, l'âge et le sexe, Monographie 1 de l'Enquête socio-économique de 2001, DGSIE, Bruxelles, 230 p. (à paraître).

Feidten P., Mulder C. H. (2002), «The Timing of Household Events and Housing Events in the Netherlands: A Longitudinal Perspective», Housing Studies, 17 (5), pp. 773-792, http://dx.doi.org/10.1080/0267303022000009808. 
Gabrielli G., Paterno A., White M. (2007), «The Impact of Origin Region and Internal Migration on Italian Fertility", Demographic Research, 17 (24), pp. 705-740, http:// dx.doi.org/10.4054/DemRes.2007.17.24.

KULU H. (2005), "Migration and Fertility: Competing Hypothesis Re-Examined», European Journal of Population, 21, pp. 51-87, http://dx.doi.org/10.1007/s10680005-3581-8.

KULU H. (2006), "Fertility of Internal Migrants: Comparison Between Austria and Poland», Population, Space and Place, 12, pp. 147-170, http://dx.doi.org/10.1002/ psp.406.

KULU H., MiLewski N. (2007), «Family Change and Migration in the Life Course: An Introduction», Demographic Research, 17 (19), pp.567-590, http://dx.doi.org/ 10.4054/DemRes.2007.17.19.

KULU H., ViKat A. (2007), «Fertility Differences by Housing Type: The Effect of Housing Conditions or of Selective Moves?», Demographic Research, 17 (26), pp. 775802, http://dx.doi.org/10.4054/DemRes.2007.17.26.

Lesthaeghe R. (1977), The Decline of Belgian Fertility, 1800-1970, Princeton University press, Princeton, $259 \mathrm{p}$.

Lesthaeghe R., Neels K. (2002), «From the First to the Second Demographic Transition - an Interpretation of the Spatial Continuity of Demographic Innovation in France, Belgium and Switzerland», European Journal of Population, 18, pp. 325360, http://dx.doi.org/10.1023/A:1021125800070.

Michielin F. (2002), "Lowest Low Fertility in an Urban Context. When Migration Plays a Key Role», MPIDR Working Paper, 50 p.

Michielin F., Mulder C. H., (2005), «Fertility Choices of Couples and Relocations in the Life Course», Paper for the IUSSP XXV International Population Conference, Tours, France, July 18-23, 21 p.

MULDeR C. H. (2006), "Population and Housing: A Two-Sided Relationship», Demographic Research, 15 (13), pp. 104-412, http://dx.doi.org/10.4054/DemRes.2006. 15.13 .

NeELS K. (2010), "Period and Cohort Effects in Belgian Nuptiality and Fertility: 19601990", Histoire de la population de la Belgique et de ses territoires, T. EGGERICKX, J.P. SANDERSON (eds), Chaire Quetelet 2005, Centre de Recherche en Démographie et Sociétés, Presses Universitaires de Louvain, Louvain-la-Neuve, pp. 463-488.

Schoenmaeckers R., Lodewijck E., Gadeyne S. (2002), Nuptialité et fécondité. Partie $B$ : fécondité, Recensement général de la population et des logements au 1er mars 1991, INS, SSTC, Bruxelles, 131 p. + annexes.

Thomas I, Vanneste D., Querriau X. (2011), Habitat. Atlas de Belgique, Gand, 78 p.

Wunsch G. (2001), "L'analyse de la fécondité par cohorte», Démographie : analyse et synthèse. I La dynamique des populations, G. CASELLI, J. VALLIN, G. WUNSCH (eds), Éditions de I'INED, Paris, pp. 149-163. 